\title{
On near optimal trajectories for a game associated with the $\infty$-Laplacian
}

\author{
Rami Atar* and Amarjit Budhiraja ${ }^{\dagger}$
}

December 1, 2008

\begin{abstract}
A two-player stochastic differential game representation has recently been obtained for solutions of the equation $-\Delta_{\infty} u=h$ in a $\mathcal{C}^{2}$ domain with Dirichlet boundary condition, where $h$ is continuous and takes values in $\mathbb{R} \backslash\{0\}$. Under appropriate assumptions, including smoothness of $u$, the vanishing $\delta$ limit law of the state process, when both players play $\delta$-optimally, is identified as a diffusion process with coefficients given explicitly in terms of derivatives of the function $u$.
\end{abstract}

AMS 2000 subject classifications: 91A15, 91A23, 35J70

Keywords: Stochastic differential games; Infinity-Laplacian; Bellman-Issacs equations

\section{Introduction and main result}

Consider the equation

$$
\begin{cases}-2 \Delta_{\infty} u=h & \text { in } G \\ u=g & \text { on } \partial G\end{cases}
$$

where, for an integer $m \geq 2, G \subset \mathbb{R}^{m}$ is a bounded $\mathcal{C}^{2}$ domain, and $g \in \mathcal{C}(\partial G, \mathbb{R})$ and the functions $h \in \mathcal{C}(\bar{G}, \mathbb{R} \backslash\{0\})$ are given. The infinity-Laplacian is defined as

$$
\Delta_{\infty} f=\frac{1}{|D f|^{2}} \sum_{i, j=1}^{m} D_{i} f D_{i j} f D_{j} f=\frac{D f^{\prime}}{|D f|} D^{2} f \frac{D f}{|D f|},
$$

provided $D f \neq 0$, where for a $\mathcal{C}^{2}$ function $f$ we denote by $D f$ the gradient and by $D^{2} f$ the Hessian matrix. We refer the reader to [1, 2, 4, 5, 6, 6, 8] for background on the infinity-Laplacian and some related PDE theory. This paper is motivated by recent work of Peres et. al. [7], where a discrete time random turn game, referred to as Tug-of-War, is developed in relation to (1.1). This game, parameterized by $\varepsilon>0$, has the property that the vanishing- $\varepsilon$ limit of the value

${ }^{*}$ Research supported in part by the Israel Science Foundation (Grant 1349/08)

${ }^{\dagger}$ Research supported in part by the Army Research Office (Grant W911NF-0-1-0080). 
function uniquely solves (1.1) in the viscosity sense (a result that is valid also in the homogenous case, $h=0$, excluded from the current paper). The stochastic differential equation (SDE)

$$
d X_{t}=2 \bar{p}\left(X_{t}\right) d W_{t}+2 q\left(X_{t}\right) d t
$$

where

$$
\bar{p}=\frac{D u}{|D u|}, \quad q=\frac{1}{|D u|^{2}}\left(D^{2} u D u-\Delta_{\infty} u D u\right),
$$

is suggested in [7] as the game's dynamics in the vanishing- $\varepsilon$ limit. The relation is rigorously established in examples, but only heuristically justified in general. In [3], a two-player zero-sum stochastic differential game (SDG) is considered, for which the value function uniquely solves (1.1) in the viscosity sense. The goal of the present paper is to show that, with appropriate conditions, (1.2) can be rigorously interpreted as the optimal dynamics of the SDG. Defined in the Elliott-Kalton sense, the SDG of [3] is formulated in such a way that one of the players selects a strategy, and then the other selects a control process (see Definition 1.1 below). We will assume in this paper that the equation possesses a classical solution $u$ i.e., $\mathcal{C}^{2}$ with nonvanishing gradient. Under this assumption we specify, for each $\delta>0$, a $\delta$-optimal strategy $\beta^{\delta}$, and a control process $Y^{\delta}$ that is $\delta$-optimal for play against $\beta^{\delta}$, in terms of first and second derivatives of $u$. We then identify the limit law, as $\delta \rightarrow 0$, of the state process under $\left(\beta^{\delta}, Y^{\delta}\right)$, as the solution $X$ to the SDE (1.2), stopped when $X$ hits the boundary $\partial G$.

A stronger result, of identifying the limit under any $\delta$-optimal play, is of interest but appears to be difficult, and is not treated in this paper.

The construction of near optimal strategy-control pairs, that may be of interest by its own right, is based on an interpretation of (1.1) as the following Bellman-Isaacs type equation (see also (2.4))

$$
\sup _{|b|=1, d \geq 0} \inf _{|a|=1, c \geq 0}\left\{-\frac{1}{2}(a-b)^{\prime}\left(D^{2} u\right)(a-b)-(c+d)(a+b) \cdot D u\right\}=h .
$$

In this form there is a natural way to construct strategy and control, by associating the supremum and infimum with the two players. The variables $a, b, c$ and $d$ selected by the players dictate the coefficients of the game's state process, and, as we prove, the coefficients converge to those of equation (1.2) in the limit as the supremum and infimum are achieved. This convergence is then lifted to the convergence of the underlying processes to the diffusion (1.2).

In the rest of this section, we describe the setting and state the main result. The proof appears in Section 2.

Throughout, we will make the following

Assumption 1.1. There exists a $\mathcal{C}^{2}(\bar{G})$ function $u$, with $D u \neq 0$ on $\bar{G}$, that solves (1.1) in the classical sense.

As a consequence of [7], that proves uniqueness (and existence) of viscosity solutions to (1.1) under the above assumptions on $h$, the function $u$ of Assumption 1.1 is the unique classical solution of (1.1). 
We now present the SDG. Let $\left(\Omega, \mathcal{F},\left\{\mathcal{F}_{t}\right\}, \boldsymbol{P}\right)$ be a complete filtered probability space with right-continuous filtration, supporting an $(m+1)$-dimensional $\left\{\mathcal{F}_{t}\right\}$-Brownian motion $\bar{W}=$ $(W, \widetilde{W})$, where $W$ and $\widetilde{W}$ are 1 - and $m$-dimensional Brownian motions, respectively. Denote by $\boldsymbol{E}$ the expectation with respect to $\boldsymbol{P}$. Let $X_{t}$ be a process taking values in $\mathbb{R}^{m}$, given by

$$
X_{t}=x+\int_{0}^{t}\left(A_{s}-B_{s}\right) d W_{s}+\int_{0}^{t}\left(C_{s}+D_{s}\right)\left(A_{s}+B_{s}\right) d s, \quad t \in[0, \infty),
$$

where $x \in \bar{G}, A_{t}$ and $B_{t}$ take values in the unit sphere $\mathcal{S}^{m-1} \subset \mathbb{R}^{m}$, and $C_{t}$ and $D_{t}$ take values in $[0, \infty)$. Denote

$$
Y^{0}=(A, C), \quad Z^{0}=(B, D) .
$$

The processes $Y^{0}$ and $Z^{0}$ take values in $\mathcal{H}=\mathcal{S}^{m-1} \times[0, \infty)$. These processes will correspond to control actions of the maximizing and minimizing player, respectively. For a process $H^{0}=$ $(A, C)$ taking values in $\mathcal{H}$ we let $S\left(H^{0}\right)=$ ess sup $\sup _{t \in[0, \infty)} C_{t}$. In the formulation below, each player initially declares a bound $S$, and then plays so as to keep $S\left(H^{0}\right) \leq S$.

Definition 1.1. (i) A pair $H=\left(\left\{H_{t}^{0}\right\}, S\right)$, where $S \in \mathbb{N}$ and $\left\{H_{t}^{0}\right\}$ is a process taking values in $\mathcal{H}$, is said to be an admissible control if $\left\{H_{t}^{0}\right\}$ is $\left\{\mathcal{F}_{t}\right\}$-progressively measurable, and $S\left(H^{0}\right) \leq S$. The set of all admissible controls is denoted by $M$. For $H=\left(\left\{H_{t}^{0}\right\}, S\right) \in M$, denote $\boldsymbol{S}(H)=S$. (ii) A mapping $\varrho: M \rightarrow M$ is said to be a strategy if, for every $t$, and $H, \widetilde{H} \in M$,

$$
\boldsymbol{P}\left(H_{s}^{0}=\widetilde{H}_{s}^{0} \text { for a.e. } s \in[0, t]\right)=1 \text { and } S=\widetilde{S}
$$

implies

$$
\boldsymbol{P}\left(I_{s}^{0}=\widetilde{I}_{s}^{0} \text { for a.e. } s \in[0, t]\right)=1 \text { and } T=\widetilde{T},
$$

where $\left(I^{0}, T\right)=\varrho\left[\left(H^{0}, S\right)\right]$ and $\left(\widetilde{I}^{0}, \widetilde{T}\right)=\varrho\left[\left(\widetilde{H}^{0}, \widetilde{S}\right)\right]$. The set of all strategies is denoted by $\widetilde{\Gamma}$. For $\varrho \in \widetilde{\Gamma}$, let $\boldsymbol{S}(\varrho)=\sup _{H \in M} \boldsymbol{S}(\varrho[H])$. Let

$$
\Gamma=\{\varrho \in \widetilde{\Gamma}: \boldsymbol{S}(\varrho)<\infty\} .
$$

Note that the Brownian motion $\widetilde{W}$ does not appear explicitly in the state dynamics, however the control processes may depend on $\widetilde{W}$. Such a formulation where the underlying filtration is rich enough to support an $(m+1)$-dimensional Brownian motion originates from Swiech's construction [10], and is crucially used in the proof of wellposedness of the SDG (see [3] for details).

We will use the symbols $Y$ and $\alpha$ for a generic control and strategy for the maximizing player, and $Z$ and $\beta$ will denote the same for the minimizing player. For a process $\xi$ taking values in $\mathbb{R}^{m}$ and a set $A \subset \mathbb{R}^{m}$, we will write $\tau_{A}(\xi)$ for

$$
\inf \left\{t \geq 0: \xi_{t} \notin A\right\}
$$

(where the infimum over an empty set is $\infty$ ). Let

$$
\tau=\tau_{G}(X)
$$


We write

$$
X\left(x, Y^{0}, Z^{0}\right) \quad\left[\text { resp., } \tau\left(x, Y^{0}, Z^{0}\right)\right]
$$

for the process $X$ [resp., the random time $\tau$ ] when it is important to specify the explicit dependence on $\left(x, Y^{0}, Z^{0}\right)$. If $\tau<\infty$ a.s., then the payoff $J\left(x, Y^{0}, Z^{0}\right)$ is well defined with values in $[-\infty, \infty]$, where

$$
J\left(x, Y^{0}, Z^{0}\right)=\boldsymbol{E}\left[\int_{0}^{\tau} h\left(X_{s}\right) d s+g\left(X_{\tau}\right)\right],
$$

and $X$ is given by (1.4). When $\boldsymbol{P}\left(\tau\left(x, Y^{0}, Z^{0}\right)=\infty\right)>0$, we set, consistent with the expectation of the first term in (1.7), $J\left(x, Y^{0}, Z^{0}\right)$ to be $+\infty[-\infty]$ for the case $h>0$ [resp., $h<0]$.

If $Y=\left(Y^{0}, K\right), Z=\left(Z^{0}, L\right) \in M$, we sometimes write $J(x, Y, Z)=J\left(x,\left(Y^{0}, K\right),\left(Z^{0}, L\right)\right)$ for $J\left(x, Y^{0}, Z^{0}\right)$. Similar conventions will be used for $X(x, Y, Z)$ and $\tau(x, Y, Z)$. Occasionally, with an abuse of terminology, when $Y=\left(Y^{0}, K\right) \in M$, we will write $Y^{0} \in M$. Let

$$
\begin{aligned}
& J^{x}(Y, \beta)=J(x, Y, \beta[Y]), \quad x \in \bar{G}, Y \in M, \beta \in \Gamma, \\
& J^{x}(\alpha, Z)=J(x, \alpha[Z], Z), \quad x \in \bar{G}, \alpha \in \Gamma, Z \in M .
\end{aligned}
$$

Define analogously $X^{x}(Y, \beta), X^{x}(\alpha, Z), \tau^{x}(Y, \beta)$ and $\tau^{x}(\alpha, Z)$ via (1.6). Define the lower value of the SDG by

$$
V(x)=\inf _{\beta \in \Gamma} \sup _{Y \in M} J^{x}(Y, \beta)
$$

and the upper value by

$$
U(x)=\sup _{\alpha \in \Gamma} \inf _{Z \in M} J^{x}(\alpha, Z) .
$$

The game is said to have value if $U=V$.

Theorem 1.1 of [3] shows that the SDG has value, and that $U=V=u$ on $\bar{G}$.

Let $x \in \bar{G}$ and $\delta>0$ be given. We say that a policy $\beta \in \Gamma$ is $\delta$-optimal for the lower game and initial condition $x$ if $\sup _{Y \in M} J^{x}(Y, \beta) \leq V(x)+\delta$. When a strategy $\beta \in \Gamma$ is given, we say that a control $Y \in M$ is $\delta$-optimal for play against $\beta$ with initial condition $x$, if $J^{x}(Y, \beta) \geq \sup _{Y^{\prime} \in M} J^{x}\left(Y^{\prime}, \beta\right)-\delta$. A pair $(Y, \beta)$ is said to be a $\delta$-optimal play for the lower game with initial condition $x$, if $\beta$ is $\delta$-optimal for the lower game and $Y$ is $\delta$-optimal for play against $\beta$ (both considered with initial condition $x$ ). Note that for such a $(Y, \beta)$ pair

$$
J^{x}(Y, \beta)-\delta \leq V(x) \leq J^{x}(Y, \beta)+\delta .
$$

An $(\alpha, Z) \delta$-optimal play for the upper game with initial condition $x$ is defined in a similar manner.

Our main result is the following. 
Theorem 1.1. Let Assumption 1.1 hold. In addition, assume there exist uniformly continuous, bounded extensions of $\bar{p}$ and $q$ to all of $\mathbb{R}^{m}$ such that, for every $x \in \mathbb{R}^{m}$, weak uniqueness holds for solutions of (1.2) starting from $x$. Fix $x \in \bar{G}$ and let $X$ and $\tau$ denote such a solution and, respectively, the corresponding exit time from $G$. Then, given any sequence $\left\{\delta_{n}\right\}_{n \geq 1}, \delta_{n} \downarrow 0$, there exists a sequence of strategy-control pairs $\left(\beta^{n}, Y^{n}\right) \in M \times \Gamma, n \geq 1$, with the following properties.

i. For every $n$, the pair $\left(\beta^{n}, Y^{n}\right)$ forms a $\delta_{n}$-optimal play for the lower game with initial condition $x$;

ii. Denoting $X^{n}=X^{x}\left(Y^{n}, \beta^{n}\right)$ and $\tau^{n}=\tau_{G}\left(X^{n}\right)$, one has that $\left(X^{n}\left(\cdot \wedge \tau^{n}\right), \tau^{n}\right)$ converges in distribution to $(X(\cdot \wedge \tau), \tau)$, as a sequence of random variables with values in $C([0, \infty)$ : $\bar{G}) \times[0, \infty]$.

An analogous result holds for the upper game.

Remark. One can always find uniformly continuous bounded extensions of $\bar{p}$ and $q$, however, in general, without additional conditions weak uniqueness may not hold. A sufficient condition for the uniqueness to hold is that $D^{2} u$ is Lipschitz on $\bar{G}$, since then both $\bar{p}$ and $q$ are Lipschitz and thus admit a Lipschitz extension to $\mathbb{R}^{m}$.

\section{Proof of the main result}

The organization of this section is as follows. We begin by recalling the Bellman-Isaacs form of (1.1), which is given in (2.4). Proposition 2.1 analyzes near maximizing and minimizing variables in (2.4). Following the construction of a strategy-control pair (that is later slightly modified, in the proof of Theorem 1.1), Proposition 2.2 proves its near optimality. Proposition 2.3 shows that, under this pair, the coefficients of the state process converge to those of (1.2). This result, along with Lemmas 2.2 2.4, is then used to prove weak convergence of the corresponding processes and exit times. The proofs of Propositions 2.1 and 2.3 appear at the end of the section.

The hypotheses of Theorem 1.1 are in force throughout this section. We will only prove the statement in Theorem 1.1 concerning the lower game. The proof for the upper game is analogous.

For $(a, c),(b, d) \in \mathcal{H}, p \in \mathbb{R}^{m}$ and $S \in \mathcal{S}(m)$ (the set of symmetric $m \times m$ matrices), let

$$
\phi(a, b, c, d ; p, S)=-\frac{1}{2}(a-b)^{\prime} S(a-b)-(c+d)(a+b) \cdot p,
$$

and denote

$$
\Lambda^{+}(p, S)=\sup _{(b, d) \in \mathcal{H}} \inf _{(a, c) \in \mathcal{H}} \phi(a, b, c, d ; p, S) .
$$

It has been shown in [3] (see Proposition 5.1 therein) that for every $p \in \mathbb{R}^{m}, p \neq 0$ and $S \in \mathcal{S}(m)$, one has

$$
\Lambda^{+}(p, S)=\Lambda(p, S):=|p|^{-2} p^{\prime} S p
$$


Throughout, we denote

$$
\begin{aligned}
& p(x)=D u(x), \quad \bar{p}(x)=\frac{p(x)}{|p(x)|}, \quad S(x)=D^{2} u(x), \\
& q(x)=\frac{1}{|p(x)|^{2}}\left(D^{2} u(x) D u(x)-\Delta_{\infty} u(x) D u(x)\right)
\end{aligned}
$$

and

$$
\psi(x, y, z)=-h(x)+\phi(a, b, c, d ; p(x), S(x)), \quad y=(a, c), z=(b, d) .
$$

Since $u$ satisfies (1.1) in the classical sense, and since $\Lambda^{+}=\Lambda$, we have

$$
\sup _{z \in \mathcal{H}} \inf _{y \in \mathcal{H}} \psi(x, y, z)=0, \quad x \in \bar{G}
$$

Identity (2.4) will be the basis for the construction of a $\delta$-optimal play for the lower game. To present the construction we first need the following result. Its proof appears at the end of the section.

Proposition 2.1. For every $\delta \in(0, \infty)$ there exist $d^{\delta} \in(0, \infty)$ and $a^{\delta}: \bar{G} \rightarrow \mathcal{S}^{m-1}$ such that the following holds.

i. For $x \in \bar{G}$, let $z^{\delta}(x) \equiv\left(b^{\delta}(x), d^{\delta}(x)\right)=\left(-\bar{p}(x), d^{\delta}\right)$. Then

$$
\inf _{y \in \mathcal{H}} \psi\left(x, y, z^{\delta}(x)\right)=\min _{y \in \mathcal{S}^{m-1} \times\{0\}} \psi\left(x, y, z^{\delta}(x)\right) \in[-\delta, 0] .
$$

Moreover, $d^{\delta} \rightarrow \infty$ as $\delta \rightarrow 0$.

ii. With $y^{\delta}(x)=\left(a^{\delta}(x), 0\right)$,

$$
\psi\left(x, y^{\delta}(x), z^{\delta}(x)\right) \in[-\delta, \delta], \quad x \in \bar{G} .
$$

Moreover, $a^{\delta}$ is Lipschitz in $x$ for every $\delta$. Finally,

$$
a^{\delta} \rightarrow \bar{p}, \quad \text { uniformly, as } \delta \rightarrow 0,
$$

and

$$
d^{\delta}\left(a^{\delta}-\bar{p}\right) \rightarrow 2 q, \quad \text { uniformly, as } \delta \rightarrow 0 .
$$

To define $\beta^{x, \delta} \equiv \beta^{\delta}$ (the dependence on the initial condition is suppressed in some instances), let $Y=\left(Y^{0}, K\right) \in M$, with $Y^{0}=(A, C)$, be given, and consider the equation

$$
d X=\left(A-b^{\delta}(X)\right) d W+\left(C+d^{\delta}(X)\right)\left(A+b^{\delta}(X)\right) d s, X_{0}=x
$$

where $b^{\delta}(x)=-\bar{p}(x)$, and $d^{\delta}(x)=d^{\delta}$. By the Lipschitz property of $b^{\delta}$, this equation has a unique solution. This defines a process $Z^{\delta}=\left(b^{\delta}(X), d^{\delta}(X)\right)$, hence a mapping, $Y \mapsto\left(Z^{\delta}, d^{\delta}\right) \in$ $M$, which is easily seen to be a strategy. This strategy will be denoted by $\beta^{\delta}$. 
Next, consider the equation

$$
d X=P^{\delta}(X) d W+Q^{\delta}(X) d s, X_{0}=x
$$

where

$$
\begin{gathered}
P^{\delta}(x)=a^{\delta}(x)-b^{\delta}(x)=a^{\delta}(x)+\bar{p}(x), \\
Q^{\delta}(x)=\left(c^{\delta}(x)+d^{\delta}(x)\right)\left(a^{\delta}(x)+b^{\delta}(x)\right)=d^{\delta}\left(a^{\delta}(x)-\bar{p}(x)\right), c^{\delta}=0 .
\end{gathered}
$$

Since the coefficients $P^{\delta}, Q^{\delta}$ are Lipschitz, there is a unique solution to (2.10). Define $\bar{Y}^{\delta, x}=$ $\bar{Y}^{\delta}=\left(a^{\delta}(X), c^{\delta}(X)\right)$. Clearly $\left(\bar{Y}^{\delta}, 1\right) \in M$ and $\beta^{\delta}\left(\bar{Y}^{\delta}, 1\right)=\left(\left(b^{\delta}(X), d^{\delta}\right), d^{\delta}\right)$.

Towards arguing that the strategy-control pair constructed above forms a nearly optimal play, we shall use the following

Lemma 2.1. For every $x \in \bar{G}, Y, Z \in M$, one has

$$
u(x)=\boldsymbol{E}\left[u\left(X_{t \wedge \tau}\right)+\int_{0}^{t \wedge \tau}\left(\psi\left(X_{s}, Y_{s}, Z_{s}\right)+h\left(X_{s}\right)\right) d s\right], \quad t \geq 0,
$$

and, if $\boldsymbol{E}[\tau]<\infty$, one has

$$
J(x, Y, Z)=V(x)-\boldsymbol{E}\left[\int_{0}^{\tau} \psi\left(X_{s}, Y_{s}, Z_{s}\right) d s\right],
$$

where $X=X(x, Y, Z)$ and $\tau=\tau_{G}(X)$.

Proof. The two identities are immediate consequences of Ito's formula applied to the smooth function $u$, the boundary condition $u=g$ on $\partial G$, and the equality $u=V$.

In what follows, let $c_{0}<\infty$ be a constant such that

$$
|h(x)|+|g(y)|+|u(x)|+|D u(x)|+\operatorname{Lip}(\bar{p}) \leq c_{0}, \quad x \in \bar{G}, y \in \partial G .
$$

Denote $\underline{h}=\inf _{x \in G}|h(x)|$.

Proposition 2.2. Fix $x \in \bar{G}$. There exist $\eta, c \in(0, \infty)$ such that for every $\delta \in(0, \eta)$, $\left(\bar{Y}^{x, \delta}, \beta^{x, \delta}\right)$ forms a $c \delta$-optimal play for the initial condition $x$.

Proof. Fix $Y=\left(Y^{0}, K\right) \in M$ with $Y^{0}=(A, C)$. Let $X$ denote the unique solution of (2.9) with this choice of $(A, C)$ and let $Z^{\delta}, d^{\delta}$ be as introduced above (2.10). Then $\beta^{\delta}(Y)=\left(Z^{\delta}, d^{\delta}\right)$. By (2.5), for every $s$,

$$
\psi\left(X_{s}, Y_{s}^{0}, Z_{s}^{\delta}\right)=\psi\left(X_{s}, Y_{s}^{0}, z^{\delta}\left(X_{s}\right)\right) \geq-\delta .
$$

Let $\eta=\underline{h} / 2$. Consider first the case $h>0$. For $\delta<\eta$, we have by (2.11)

$$
\boldsymbol{E}[t \wedge \tau] \leq c_{1}:=4 \underline{h}^{-1} c_{0},
$$

and consequently $\boldsymbol{E}[\tau] \leq c_{1}$, where $\tau=\tau^{x}\left[Y, \beta^{\delta}\right]$. Hence using (2.14) in (2.12),

$$
J^{x}\left(Y, \beta^{\delta}\right) \leq V(x)+\delta \boldsymbol{E}[\tau] \leq V(x)+c_{1} \delta .
$$


Since $Y \in M$ is arbitrary, this shows that $\beta^{\delta}$ is $c_{1} \delta$-optimal.

Consider now the case $h<0$. Fix $e \in \mathcal{S}^{m-1}$ and let $(e, 1)=\tilde{Y} \in M$. It is easily checked (see Lemma 3.1 of [3]) that $\inf _{\beta \in \Gamma} J^{x}(\widetilde{Y}, \beta)>-\infty$. Thus $\inf _{\delta} C\left(\beta^{\delta}\right):=\underline{c}>-\infty$, where for $\beta \in \Gamma, C(\beta)=\sup _{Y \in M} J^{x}(Y, \beta)$. Let $M_{\delta}=\left\{Y \in M: J^{x}\left(Y, \beta^{\delta}\right)>\underline{c}-1\right\}$. Then $C(\beta)=$ $\sup _{Y \in M_{\delta}} J^{x}(Y, \beta)$. Note that for $Y \in M_{\delta}, \tau=\tau^{x}\left(Y, \beta^{\delta}\right)<\infty$ a.s. and

$$
\underline{c}-1<J^{x}\left(Y, \beta^{\delta}\right) \leq-\underline{h} \boldsymbol{E}[\tau]+c_{0} .
$$

Thus for the case $h<0$ as well, $\beta^{\delta}$ is $c_{2} \delta$-optimal, for some $c_{2} \in(0, \infty)$.

Recall that $\bar{Y}_{s}^{\delta}=y^{\delta}\left(X_{s}\right)$, where $X$ is the unique solution of (2.10) and note that $\beta^{\delta}\left(\left(\bar{Y}^{\delta}, 1\right)\right)=$ $\left(Z^{\delta}(X), d^{\delta}\right)$. By (2.6),

$$
\psi\left(X_{s}, y^{\delta}\left(X_{s}\right), z^{\delta}\left(X_{s}\right)\right) \leq \delta .
$$

Observing that $\boldsymbol{E}[\tau] \leq c_{1}$, where $\tau=\tau^{x}\left(\bar{Y}^{\delta}, \beta^{\delta}\right)$, we have using (2.16) in (2.12),

$$
J^{x}\left(\bar{Y}^{\delta}, \beta^{\delta}\right) \geq V(x)-c_{1} \delta \geq \sup _{Y \in M} J^{x}\left(Y, \beta^{\delta}\right)-2\left(c_{1} \vee c_{2}\right) \delta
$$

where the last inequality follows from the $\left(c_{1} \vee c_{2}\right) \delta$-optimality of $\beta^{\delta}$. The result follows.

The proof of the following proposition is given towards the end of the section. Denote by $p^{*}$ and $q^{*}$ the continuous, bounded extensions of $\bar{p}$ and $q$ to $\mathbb{R}^{m}$, satisfying the hypotheses of Theorem 1.1 .

Proposition 2.3. Let $\left\{\delta_{n}\right\}_{n \geq 1}$ be a sequence in $\mathbb{R}_{+}$such that $\delta_{n} \rightarrow 0$ as $n \rightarrow \infty$. Then there exists a sequence of (open) domains $G_{n-1} \subset \subset G_{n} \subset G, G_{n} \uparrow G$ as $n \rightarrow \infty$ and continuous, uniformly bounded maps $p_{n}^{*}, q_{n}^{*}$ from $\mathbb{R}^{m}$ to itself, $p_{n}^{*} \rightarrow p^{*}, q_{n}^{*} \rightarrow q^{*}$, uniformly on $\mathbb{R}^{m}$, such that $p_{n}^{*}=\bar{p}_{n}$ and $q_{n}^{*}=\bar{q}_{n}$ on $G_{n}$, where $\bar{p}_{n}=\frac{1}{2}\left(a^{\delta_{n}}+\bar{p}\right)$ and $\bar{q}_{n}=\frac{1}{2} d^{\delta_{n}}\left(a^{\delta_{n}}-\bar{p}\right)$.

Lemma 2.2. With notation as in Proposition 2.3, let $\bar{X}_{n}, \bar{X}$ be solutions of

$$
d \bar{X}_{n}=2 p_{n}^{*}\left(\bar{X}_{n}\right) d W+2 q_{n}^{*}\left(\bar{X}_{n}\right) d t, \quad d \bar{X}=2 p^{*}(\bar{X}) d W+2 q^{*}(\bar{X}) d t,
$$

respectively, starting from $x$, and given on suitable filtered probability spaces. Denote

$$
\bar{\tau}(n, k)=\tau_{G_{k}}\left(\bar{X}^{n}\right), \quad \bar{\tau}(k)=\tau_{G_{k}}(\bar{X}), \quad \bar{\tau}=\tau_{G}(\bar{X}) .
$$

Then there exists a sequence $\left\{\ell_{n}\right\}_{n \geq 1}, \ell_{n} \uparrow \infty$ as $n \rightarrow \infty$, such that

$$
\left(\bar{X}_{n}\left(\cdot \wedge \bar{\sigma}_{n}\right), \bar{\sigma}_{n}\right) \Rightarrow(\bar{X}(\cdot \wedge \bar{\tau}), \bar{\tau})
$$

as a sequence of $C([0, \infty): \bar{G}) \times[0, \infty]$-valued random variables, where $\bar{\sigma}_{n}=\bar{\tau}\left(n, \ell_{n}\right)$.

Proof. The coefficients $p_{n}^{*}$ and $q_{n}^{*}$ converge uniformly on $\mathbb{R}^{m}$ to $p^{*}$ and $q^{*}$, respectively, by Proposition 2.3. Moreover, by assumption, weak uniqueness holds for solutions to the SDE associated with $\left(p^{*}, q^{*}\right)$, starting from $x$, for any $x \in \mathbb{R}^{m}$. Thus Theorem 11.1.4 of [9] is in force, and we can deduce that $\bar{X}^{n}$ converges to $\bar{X}$ in distribution, as $n \rightarrow \infty$. 
We now assume without loss of generality that $\bar{X}_{n}, \bar{X}$ are given on a common probability space and $\bar{X}_{n} \rightarrow \bar{X}$, a.s., in $C([0, \infty))$. For $t>0$ let $E_{t}=\{\omega: \bar{\tau}(\omega) \leq t\}$. Fix $\omega \in E_{t}$. Given $k \in \mathbb{N}$, choose $\delta>0$ such that $\left|y_{1}-y_{2}\right|>\delta$ for all $y_{1} \in G_{k}, y_{2} \in \partial G$. Let $n_{0}=n_{0}(\delta, t, \omega)$ be such that, for all $n \geq n_{0},\left|\bar{X}_{n}-\bar{X}\right|_{t}^{*}<\delta$. Note that $\bar{X}(\bar{\tau}(\omega)) \in \partial G$ and so $\bar{X}_{n}(\bar{\tau}(\omega)) \notin G_{k}$. In particular, $\bar{\tau}(n, k)(\omega) \leq \bar{\tau}(\omega)$. Letting $n \rightarrow \infty$, we get $\lim _{\sup _{n \rightarrow \infty}} \bar{\tau}(n, k) \leq \bar{\tau}$, for all $\omega \in E_{t}$.

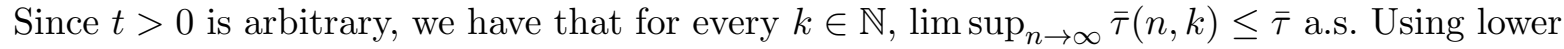
semi-continuity property of exit times we then have a.s.,

$$
\bar{\tau}(k) \leq \liminf _{n \rightarrow \infty} \bar{\tau}(n, k) \leq \limsup _{n \rightarrow \infty} \bar{\tau}(n, k) \leq \bar{\tau} .
$$

Also note that $\bar{\tau}(k) \rightarrow \bar{\tau}$ a.s., as $k \rightarrow \infty$.

Let $F=\{\bar{\tau}<\infty\}$. In what follows, for an event $E$, we will write $\boldsymbol{P}(E F)$ as $\boldsymbol{P}_{F}(E) . \boldsymbol{P}_{F^{c}}$ is defined similarly. From the above display we have that for every $\varepsilon>0$

$$
\limsup _{k \rightarrow \infty} \limsup _{n \rightarrow \infty} \boldsymbol{P}_{F}(|\bar{\tau}(n, k)-\bar{\tau}|>\varepsilon)=0 .
$$

We can then find a sequence $\{\varepsilon(k)\}_{k \geq 1}, \varepsilon(k) \in(0, \infty)$ such that $\varepsilon(k) \downarrow 0$ as $k \rightarrow \infty$ and

$$
\limsup _{k \rightarrow \infty} \limsup _{n \rightarrow \infty} \boldsymbol{P}_{F}(|\bar{\tau}(n, k)-\bar{\tau}|>\varepsilon(k))=0 .
$$

Finally, choose a sequence $\left\{\ell_{n}\right\}_{n \geq 1}$ such that $\ell_{n} \uparrow \infty$ as $n \rightarrow \infty$ and

$$
\lim _{n \rightarrow \infty} \boldsymbol{P}_{F}\left(\left|\bar{\tau}\left(n, \ell_{n}\right)-\bar{\tau}\right|>\varepsilon\left(\ell_{n}\right)\right)=0 .
$$

In a similar fashion, by choosing a further subsequence if needed, we have that for every $r>0$

$$
\lim _{n \rightarrow \infty} \boldsymbol{P}_{F^{c}}\left(\bar{\tau}\left(n, \ell_{n}\right) \leq r\right)=0 .
$$

Combining the above displays we have $\bar{\sigma}_{n}=\bar{\tau}\left(n, \ell_{n}\right) \rightarrow \bar{\tau}$ in probability as $n \rightarrow \infty$. The result follows.

Lemma 2.3. Let $X^{n}$ be the (pathwise) unique solution of (2.10) with $\delta=\delta_{n}$ (stopped when the boundary is reached). Let $\left(G_{n}, p_{n}^{*}, q_{n}^{*}\right)_{n \geq 1}, p^{*}, q^{*}$ be as in Proposition 2.3 and $\left\{\ell_{n}\right\}_{n \geq 1}$ be as in Lemma 2.2. Let $X$ solve (1.2) with initial condition $x$. Then

$$
\left(X^{n}\left(\cdot \wedge \eta_{n}\right), \eta_{n}\right) \Rightarrow(X(\cdot \wedge \tau), \tau)
$$

where $\eta^{n}=\tau_{G^{n \wedge \ell_{n}}}\left(X^{n}\right)$ and $\tau=\tau_{G}(X)$.

Proof. Let $\bar{X}^{n}, \bar{X}$ be as in Lemma 2.2. Then from Proposition $2.3(\bar{X}(\cdot \wedge \bar{\tau}), \bar{\tau})$ has the same law as $(X(\cdot \wedge \tau), \tau)$ and $\left(\bar{X}^{n}\left(\cdot \wedge \bar{\eta}_{n}\right), \bar{\eta}_{n}\right)$ has the same law as $\left(X^{n}\left(\cdot \wedge \eta_{n}\right), \eta_{n}\right)$, where $\bar{\eta}^{n}$ is defined similarly to $\eta^{n}$ by replacing $X^{n}$ with $\bar{X}^{n}$. By lower semi-continuity of exit times, $\liminf _{n} \bar{\tau}(n, n) \geq \bar{\tau}$ a.s. The result now follows from Lemma 2.2 on noting that $\bar{\eta}^{n}=\bar{\tau}(n, n) \wedge$ $\bar{\sigma}^{n}$. 
Lemma 2.4. Let $X$ be a solution of (1.2) given on some filtered probability space, with $X_{0}=$ $x \in \bar{G}$. Let $\tau=\tau_{G}(X)$. Then $\boldsymbol{E}[\tau]<\infty$ and

$$
u(x)=\boldsymbol{E}\left[g\left(X_{\tau}\right)+\int_{0}^{\tau} h\left(X_{s}\right) d s\right] .
$$

Proof. Applying Itô's formula to $u(X)$ and recalling that $u$ is a classical solution of (1.1), we obtain

$$
u(x)=\boldsymbol{E}\left[u\left(X_{\tau \wedge t}\right)+\int_{0}^{\tau \wedge t} h\left(X_{s}\right) d s\right],
$$

for every $t>0$. The property $\boldsymbol{E}[\tau]<\infty$ is now immediate on recalling that $h$ is either positive or negative, and bounded away from zero. The result follows on sending $t \rightarrow \infty$.

Proof of Theorem 1.1. Fix $x \in \bar{G}$. Let $\left\{X^{n}, G_{n}, p_{n}^{*}, q_{n}^{*}, \ell_{n}, \eta_{n}\right\}$ be as in Lemma 2.3. Let $\left(\bar{Y}^{n}, \beta^{n}\right)=\left(\bar{Y}^{x, \delta_{n}}, \beta^{x, \delta_{n}}\right)$ where, for $\delta>0,\left(\bar{Y}^{x, \delta}, \beta^{x, \delta}\right)$ is as in Proposition 2.2. Note that $X^{n}=X^{x}\left(\bar{Y}^{n}, \beta^{n}\right)$. We assume, without loss of generality, that $\delta_{n}<\underline{h} / 2$ for $n \geq 1$. Then, as in the proof of Proposition 2.2, we deduce that

$$
\boldsymbol{E}\left(\eta_{n}\right) \leq \boldsymbol{E}\left(\tau_{G}\left(X^{n}\right)\right) \leq 4 \underline{h}^{-1} c_{0},
$$

where $c_{0}$ was introduced in (2.13). From Lemma 3.2 of [3] , there exist $\widetilde{Y}^{n} \in M$ and $\left\{\delta_{n}^{1}\right\}_{n \geq 1}$, $\delta_{n}^{1} \downarrow 0$, such that $\widetilde{Y}_{t \wedge \eta_{n}}^{n}=\bar{Y}_{t \wedge \eta_{n}}^{n}$ and

$$
\boldsymbol{E}\left\{\widetilde{\tau}_{n}-\eta_{n} \mid \mathcal{F}_{\eta_{n}}\right\} \leq \delta_{n}^{1}, \boldsymbol{E}\left\{\left|\widetilde{X}^{n}-X^{n}\left(\eta_{n}\right)\right|_{*}^{2} \mid \mathcal{F}_{\eta_{n}}\right\} \leq \delta_{n}^{1},
$$

where $\widetilde{X}^{n}=X^{x}\left(\widetilde{Y}^{n}, \beta^{n}\right), \widetilde{\tau}^{n}=\tau^{x}\left(\widetilde{Y}^{n}, \beta^{n}\right)$ and $\left|\widetilde{X}^{n}-X^{n}\left(\eta_{n}\right)\right|_{*}=\sup _{t \in\left[\eta_{n}, \tilde{\tau}_{n}\right]}\left|\widetilde{X}^{n}(t)-X^{n}\left(\eta_{n}\right)\right|$.

Recall that $\beta^{n}$ is $c \delta_{n}$-optimal. We now show that $\tilde{Y}^{n}$ is $\delta_{n}^{*}$-optimal for play against $\beta^{n}$, for some sequence $\delta_{n}^{*} \rightarrow 0$. From Lemma 2.3 and (2.17) we have that

$$
\left|\boldsymbol{E}\left[\int_{0}^{\eta_{n}} h\left(X^{n}(s)\right) d s+V\left(X_{\eta_{n}}^{n}\right)\right]-\boldsymbol{E}\left[\int_{0}^{\tau} h(X(s)) d s+V\left(X_{\tau}\right)\right]\right|=\delta_{n}^{2} \rightarrow 0, \text { as } n \rightarrow \infty .
$$

From (2.18)

$$
\left|\boldsymbol{E}\left[\int_{0}^{\widetilde{\tau}_{n}} h\left(\widetilde{X}^{n}(s)\right) d s+V\left(\tilde{X}_{\widetilde{\tau}_{n}}^{n}\right)\right]-\boldsymbol{E}\left[\int_{0}^{\eta_{n}} h\left(X^{n}(s)\right) d s+V\left(X_{\eta_{n}}^{n}\right)\right]\right|=\delta_{n}^{3} \rightarrow 0, \text { as } n \rightarrow \infty .
$$

Setting $\delta_{n}^{*}=\delta_{n}^{2}+\delta_{n}^{3}+c \delta_{n}$, we have on combining the above two displays

$$
\begin{aligned}
J^{x}\left(\widetilde{Y}^{n}, \beta^{n}\right) & =\boldsymbol{E}\left[\int_{0}^{\widetilde{\tau}_{n}} h\left(\widetilde{X}_{s}^{n}\right) d s+g\left(\widetilde{X}^{n}\left(\widetilde{\tau}_{n}\right)\right)\right] \\
& \geq \boldsymbol{E}\left[\int_{0}^{\tau} h\left(X_{s}\right) d s+V(X(\tau))\right]-\left(\delta_{n}^{2}+\delta_{n}^{3}\right) \\
& =V(x)-\left(\delta_{n}^{2}+\delta_{n}^{3}\right) \\
& \geq \sup _{Y \in M} J^{x}\left(Y, \beta^{n}\right)-\delta_{n}^{*},
\end{aligned}
$$


where the equality in the third line above follows from Lemma 2.4 and the last inequality is a consequence of $c \delta_{n}$-optimality of $\beta^{n}$. Finally, from (2.18) $\sup _{0 \leq t<\infty}\left|X^{n}\left(t \wedge \eta_{n}\right)-\widetilde{X}^{n}\left(t \wedge \widetilde{\tau}_{n}\right)\right| \rightarrow 0$ and $\left|\eta_{n}-\widetilde{\tau}_{n}\right| \rightarrow 0$ in probability as $n \rightarrow \infty$. Thus, from Lemma $2.3\left(\widetilde{X}^{n}\left(\cdot \wedge \widetilde{\tau}_{n}\right), \widetilde{\tau}_{n}\right) \Rightarrow$ $(X(\cdot \wedge \tau), \tau)$ and the result follows.

Proof of Proposition 2.3. For $d \in \mathbb{N}$, let $f_{n}, f: \bar{G} \rightarrow \mathbb{R}^{d}$ be uniformly bounded continuous maps such that $f_{n} \rightarrow f$ uniformly on $\bar{G}$. Let $F: \mathbb{R}^{m} \rightarrow \mathbb{R}^{d}$ be a uniformly continuous bounded extension of $f$. Consider a sequence $\left\{E_{n}\right\}$ of (open) domains with $E_{n-1} \subset \subset E_{n} \subset G, E_{n} \uparrow G$ as $n \rightarrow \infty$. We will show that there is a collection of uniformly bounded, continuous maps $\left\{F_{n}^{k}: n \geq 1, k \geq 1\right\}$ such that $F_{n}^{k}$ agrees with $f_{n}$ on $E_{k}$ and along some subsequence $\left\{k_{n}\right\}_{n \geq 1}$, $F_{n}^{k_{n}} \rightarrow F$, uniformly on $\mathbb{R}^{m}$. The result will then follow, on setting $F=\left(p^{*}, q^{*}\right), f=(\bar{p}, q)$, $f_{n}=\left(\bar{p}_{n}, \bar{q}_{n}\right)^{\prime}$, with $G_{n}=E_{k_{n}}$.

Define

$$
\widetilde{F}_{n}(x)=f_{n}(x) 1_{x \in \bar{G}}+F(x) 1_{x \in \mathbb{R}^{m} \backslash \bar{G}} .
$$

Let $\psi$ be a $C^{\infty}$ function on $\mathbb{R}^{d}$ such that $0 \leq \psi(x) \leq 1, \operatorname{supp}(\psi) \subset B_{1}(0)$ and $\int_{\mathbb{R}^{d}} \psi(x) d x=1$, where $B_{r}(0)$ is the ball of radius $r$ in $\mathbb{R}^{m}$, centered at 0 . Let $\psi_{k}(x)=k^{m} \psi(k x)$. Define

$$
\bar{F}_{n}^{k}(x)=\int_{\mathbb{R}^{m}} \widetilde{F}_{n}(x-y) \psi_{k}(y) d y, \quad \bar{F}^{k}(x)=\int_{\mathbb{R}^{m}} F(x-y) \psi_{k}(y) d y, \quad x \in \mathbb{R}^{m} .
$$

Let $\rho^{k} \in C^{\infty}\left(\mathbb{R}^{m}\right)$ be such that $0 \leq \rho^{k}(x) \leq 1$ and

$$
\rho^{k}(x)=\left\{\begin{array}{cc}
1 & \text { if } x \in E_{k} \\
0 & \text { if } x \in G^{c} .
\end{array}\right.
$$

Define

$$
F_{n}^{k}(x)=\rho^{k}(x) f_{n}(x)+\left(1-\rho^{k}(x)\right) \bar{F}_{n}^{k}(x), \quad x \in \mathbb{R}^{m}
$$

and

$$
F^{k}(x)=\rho^{k}(x) F(x)+\left(1-\rho^{k}(x)\right) \bar{F}^{k}(x), \quad x \in \mathbb{R}^{m} .
$$

Note that $F_{n}^{k}(x)=f_{n}(x)$ and $F^{k}(x)=f(x)$ for $x \in E_{k}$ and

$$
F_{n}^{k}(x)-F^{k}(x)=\rho^{k}(x)\left(f_{n}(x)-f(x)\right)+\left(1-\rho^{k}(x)\right)\left(\bar{F}_{n}^{k}(x)-\bar{F}^{k}(x)\right), \quad x \in \mathbb{R}^{m} .
$$

Also

$$
\sup _{x \in \mathbb{R}^{m}} \sup _{k \geq 1}\left|\bar{F}_{n}^{k}(x)-\bar{F}^{k}(x)\right| \leq \sup _{x \in \bar{G}}\left|f_{n}(x)-f(x)\right| \rightarrow 0, \text { as } n \rightarrow \infty .
$$

Combining the above two displays

$$
\sup _{x \in \mathbb{R}^{m}} \sup _{k \geq 1}\left|F_{n}^{k}(x)-F^{k}(x)\right| \rightarrow 0, \text { as } n \rightarrow \infty .
$$

Next note that $\sup _{x \in \mathbb{R}^{m}}\left|\bar{F}^{k}(x)-F(x)\right| \rightarrow 0$, as $k \rightarrow \infty$ and therefore

$$
\sup _{x \in \mathbb{R}^{m}}\left|F^{k}(x)-F(x)\right| \rightarrow 0, \text { as } k \rightarrow \infty .
$$


Using the above two displays, we can find a sequence $\left\{k_{n}\right\}$ such that $F_{n}^{k_{n}} \rightarrow F$ uniformly on $\mathbb{R}^{m}$. By construction $F_{n}^{k}$ agrees with $f_{n}$ on $E_{k}$. The result follows.

Proof of Proposition 2.1. We begin by constructing functions $a^{\delta}$ for which all conclusions of the proposition hold, save the Lipschitz property. We will then argue that one can find a Lipschitz regularization of each $a^{\delta}$, for which all conclusions are still valid.

With $b=b(x)=-\bar{p}(x)$, the second term of (2.1) takes the form $(c+d)(1-a \cdot \bar{p}(x))|p(x)| \geq 0$, and therefore the infimum of $\psi(x,(a, c),(b(x), d))$, over $c$, is attained at $c=0$. The function $a \mapsto \psi(x,(a, 0),(-\bar{p}(x), d))$ is continuous, and thus the minimum over $\mathcal{S}^{m-1}$ is attained. For an arbitrary choice of $d^{\delta}$, we have by (2.4),

$$
\gamma^{\delta}(x):=\min _{y \in \mathcal{S}^{m-1} \times\{0\}} \psi\left(x, y, z^{\delta}(x)\right) \leq 0 .
$$

Later in the proof it is shown that for a suitable choice of $d^{\delta}, \gamma^{\delta}(x) \geq-\delta$ for all $x \in \bar{G}$.

For each $\delta$ and $x$ let $a^{\delta}(x)$ be a minimizer of $a \mapsto \psi\left(x,(a, 0),\left(-\bar{p}(x), d^{\delta}\right)\right)$ over $\mathcal{S}^{m-1}$. Write $y^{\delta}(x)=\left(a^{\delta}(x), 0\right)$. From (2.19) $\gamma^{\delta}(x)=\psi\left(x, y^{\delta}(x), z^{\delta}(x)\right) \leq 0$.

We show now that for any choice of $d^{\delta}$ such that $d^{\delta} \rightarrow \infty$ as $\delta \rightarrow 0$,

$$
a^{\delta}(x) \rightarrow \bar{p}(x) \text { as } \delta \rightarrow 0 \text {, uniformly in } x .
$$

Assuming the contrary, there exists $\varepsilon>0$ and, for every $\delta>0, x_{\delta} \in \bar{G}$, such that

$$
\left|a^{\delta}\left(x_{\delta}\right)-\bar{p}\left(x_{\delta}\right)\right|>\varepsilon
$$

However, because of the upper bound on $\gamma^{\delta}$, it follows that

$$
d^{\delta}\left(1-a^{\delta}(x) \cdot \bar{p}(x)\right)|p(x)| \leq c_{1},
$$

for some constant $c_{1}$ not depending on $x$ and $\delta$. This contradicts (2.21) and thus (2.20) follows. Henceforth we will assume that $d^{\delta} \rightarrow \infty$ as $\delta \rightarrow 0$.

Since $y^{\delta}$ is a minimizer, we have that $\psi\left(x, y^{\delta}(x), z^{\delta}(x)\right) \leq \psi\left(x,(\bar{p}(x), 0), z^{\delta}(x)\right)$. Along with the uniform convergence in (2.20), this implies

$$
\limsup _{\delta \rightarrow 0} \sup _{x} d^{\delta}\left(1-a^{\delta}(x) \cdot \bar{p}(x)\right)|p(x)| \leq 0 .
$$

Consequently,

$$
d^{\delta}\left(1-a^{\delta}(x) \cdot \bar{p}(x)\right) \rightarrow 0 \text { as } \delta \rightarrow 0 \text {, uniformly in } x
$$

We next show that

$$
Q^{\delta}(x):=d^{\delta}\left(a^{\delta}(x)-\bar{p}(x)\right) \rightarrow 2 q(x) \text { as } \delta \rightarrow 0, \text { uniformly in } x .
$$


Denote by $\widetilde{\phi}$ the map $a \mapsto \phi\left(a, b(x), 0, d^{\delta} ; p(x), S(x)\right)$. By the Lagrange multipliers theorem, every $a \in \mathcal{S}^{m-1}$, which minimizes $\widetilde{\phi}(a)$ satisfies $D \widetilde{\phi}(a)+\lambda a=0$ for some $\lambda \in \mathbb{R}$. Thus by definition of $a^{\delta}(x)$, suppressing the dependence on $\delta$ and $x$,

$$
\begin{gathered}
\lambda a=S(a+\bar{p})+d p, \\
\lambda=a^{\prime} S(a+\bar{p})+d a \cdot p .
\end{gathered}
$$

Hence

$$
\begin{aligned}
Q & =d(a-\bar{p})=d a-\frac{d p}{|p|} \\
& =d a-\frac{a}{|p|} \lambda+\frac{S(a+\bar{p})}{|p|} \\
& =d(1-a \cdot \bar{p}) a-\frac{a}{|p|} a^{\prime} S(a+\bar{p})+\frac{S(a+\bar{p})}{|p|} \rightarrow-\frac{2 \bar{p}}{|p|} \bar{p}^{\prime} S \bar{p}+\frac{2}{|p|} S \bar{p}=2 q,
\end{aligned}
$$

where the convergence is uniform, and we have used (2.20) and (2.22) on the last line. This shows (2.23).

We now estimate $\gamma^{\delta}$. Suppressing $x$ and $\delta$,

$$
\gamma=\psi(x, y, z)=-h-\frac{1}{2}(a+\bar{p})^{\prime} S(a+\bar{p})-d(a-\bar{p}) \cdot p .
$$

The second term converges uniformly to $-2 \bar{p}^{\prime} S \bar{p}$ which equals $h$ by (1.1), while the last term converges to zero by (2.22). Consequently we may, and will, choose $d^{\delta}$ to grow sufficiently fast so that, for every $\delta \in\left(0, \delta_{0}\right)$,

$$
\inf _{x} \gamma^{\delta}(x) \geq-\frac{\delta}{2}
$$

We now show that, for $\delta<\delta_{0}$ sufficiently small, $a^{\delta}$ is continuous. The proof is based on (2.24) and (2.25). We will suppress $\delta$ from notation unless needed.

For $i=1,2$ let $x_{i} \in \bar{G}$. Let $p_{i}=p\left(x_{i}\right)$, and similarly define the quantities $\bar{p}_{i}, S_{i}, a_{i}$ and $\lambda_{i}$, $i=1,2$. Let $\Delta p=p_{1}-p_{2}$, and similarly define $\Delta \bar{p}, \Delta S, \Delta a$ and $\Delta \lambda$. By (2.24) and (2.25),

$$
\begin{gathered}
\Delta \lambda a_{1}+\lambda_{2} \Delta a=\Delta S\left(a_{1}+\bar{p}_{1}\right)+S_{2}(\Delta a+\Delta \bar{p})+d \Delta p \\
\Delta \lambda=\Delta a^{\prime} S_{1}\left(a_{1}+\bar{p}_{1}\right)+a_{2}^{\prime} \Delta S\left(a_{1}+\bar{p}_{1}\right)+a_{2}^{\prime} S_{2}(\Delta a+\Delta \bar{p})+d \Delta a \cdot p_{1}+d a_{2} \cdot \Delta p .
\end{gathered}
$$

Thus, with $|\Delta|=\max \{|\Delta p|,|\Delta \bar{p}|,|\Delta S|\}$, and with $c_{1}, c_{2}$ independent of $\delta$,

$$
\begin{aligned}
\left|\lambda_{2}\right||\Delta a| & \leq|\Delta \lambda|+c_{1}|\Delta|+c_{1}|\Delta a|+d|\Delta| \\
& \leq c_{2}|\Delta|+c_{2}|\Delta a|+d\left|\Delta a \cdot \bar{p}_{1}\right|\left|p_{1}\right|+2 d|\Delta| .
\end{aligned}
$$

By (2.25) and uniform convergence of $a$ to $\bar{p}$, one can find a constant $c_{3}>0$ and a constant $\delta_{1} \in\left(0, \delta_{0}\right)$, such that for all $\delta<\delta_{1}$, one has $\lambda_{2}^{\delta}>c_{3} d^{\delta}, d^{\delta} \in\left(\frac{4 c_{2}}{c_{3}}, \infty\right)$, and

$$
\sup _{x}\left|a^{\delta}(x)-\bar{p}(x)\right|\left|p_{1}(x)\right| \leq c_{3} / 4 \text {. }
$$


Note that

$$
\left|\Delta a \cdot \bar{p}_{1}\right| \leq \frac{1}{2}|\Delta a|\left|a_{1}+a_{2}-2 \bar{p}_{1}\right| \leq \frac{1}{2}|\Delta a|\left|a_{1}+a_{2}-\bar{p}_{1}-\bar{p}_{2}\right|+|\Delta| .
$$

Thus for all $\delta<\delta_{1}$,

$$
c_{3}|\Delta a| \leq \frac{c_{2}}{d}(|\Delta|+|\Delta a|)+\frac{c_{3}}{4}|\Delta a|+c_{4}|\Delta| .
$$

Consequently, for all $\delta \leq \delta_{1},|\Delta a| \leq \frac{2}{c_{3}}\left(c_{3}+c_{4}\right)|\Delta|$. The continuity of $a^{\delta}$ follows.

Finally, the functions $a^{\delta}$ need not be Lipschitz in $x$. However, using a straightforward mollification argument, given $\varepsilon>0$, one can find a Lipschitz function $a^{\delta, \varepsilon}$, with values in $\mathcal{S}^{m-1}$, that is $\varepsilon$-close to $a^{\delta}$ in the uniform topology. It is possible to then let $\varepsilon$ depend on $\delta$ in such a way that $\hat{a}^{\delta}:=a^{\delta, \varepsilon(\delta)}$ satisfy results analogous to (2.20), (2.22) and (2.23). Furthermore, using (2.19) and (2.26), one can ensure that $\hat{a}^{\delta}$ satisfies (2.6). This completes the proof.

\section{References}

[1] G. Aronsson. Extension of functions satisfying Lipschitz conditions. Ark. Mat. 6, 551-561 (1967)

[2] G. Aronsson. A mathematical model in sand mechanics: presentation and analysis. SIAM J. Appl. Math., 22 (1972), 437-458

[3] R. Atar and A. Budhiraja. A stochastic differential game for the inhomogeneous $\infty$ Laplace equation. Preprint, Math. arXiv:0808.1457.

[4] E. N. Barron, L. C. Evans and R. Jensen. The infinity Laplacian, Aronsson's equation and their generalizations. Preprint

[5] R. Jensen. Uniqueness of Lipschitz extensions: Minimizing the sup norm of the gradient. Arch. Rational Mech. Anal., 123(1):5174, 1993.

[6] R. V. Kohn and S. Serfaty. A deterministic-control-based approach to motion by curvature. Comm. Pure Appl. Math. 59 (2006), no. 3, 344-407

[7] Y. Peres, O. Schramm, S. Sheffield and D. B. Wilson. Tug-of-war and the infinity Laplacian. Jour. AMS, to appear

[8] H. M. Soner and N. Touzi. A stochastic representation for mean curvature type geometric flows. Ann. Probab. 31 (2003), no. 3, 1145-1165

[9] D. W. Stroock and S. R. S. Varadhan. Multidimensional Diffusion Processes. SpringerVerlag, Berlin, 2006

[10] A. Swiech. Another approach to the existence of value functions of stochastic differential games. J. Math. Anal. Appl. 204 (1996), no. 3, 884-897

Department of Electrical Engineering

Technion-IsRael Institute of Technology

HAIFA 32000, ISRAEL

Department of Statistics and Operations Research

University of North Carolina

Chapel Hill, NC 27599, USA 